\title{
CORRESPONDENCE/
}

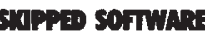

To the editor:

Tn an article by Pamela Knight entiIled "Microcomputer Software Compares 2-D Gels," (Bio/Technology 6:999, Sept. '88) Bio-Rad's 2-D Analyst II software for comparison of 2-D gels was not mentioned. We would like to point out that this system provides $2-D$ quantitative analyses, including automatic gel to gel comparisons and single spot analysis.

Douglas Patterson Marketing Specialist Electrophoresis Business Unit Bio-Rad Laboratories

1414 Harbour Way South Richmond, CA 94804

\section{Fun may}

To the editor:

$T$ read with interest Jennifer $\mathrm{Van}$ Brunt's report "Molecular Farming: Transgenic Animals as Bioreactors" (Bio/Technology 6:1 149, Oct. '88) and was quite surprised that she left out our important work on the production of virus-resistant transgenic chickens. (See Salter, D. W. and Crittenden, L. B. Science 66:170, 1987, for example.) In addition, our work has also been cited in the news in 1987 and 1988.

Donald Wayne Salter, Ph.D. Assistant Research Professor Dept. of Microbiology and Public Health Michigan State University East Lansing, MI 48824

\section{To the editor:}

A s one of the leading manufacturAlers of fermentation equipment worldwide, we at B. Braun Diessel Biotech $\mathrm{GmbH}$ were dismayed at the omission of our products from the chart listing pilot-scale fermentors and bioreactors in the special report on fermentation by Pamela Knight (Bio/Technology 6:505, May '88).

We would like to inform your read- ers that our products include the Biostat $^{\circledR}$ fermentors, which are well known as state-of-the-art for flexible applications in both laboratory- and pilot-scale. Special cell culture fermentors are available with reactor design and equipment adapted for bubble-free aeration, perfusion, etc.

This year, we have launched a new generation of laboratory fermentors with digital control. This technique allows automation of fermentors with control strategies, direct computer connections, and easy operation.

Dr. Dziengel and Dr. Martin

B. Braun Diessel Biotech $\mathrm{GmbH}$ P.O. Box 120

D-3508 Melsungen West Grermany

\section{A Lason Fin O4}

To the editor:

Thank you for your column on the 1 HIV CD4 protein "mistake"(Bio/ Technology 6:1268, Nov. '88). I have used this example in my elementary genetics class and also forwarded the column to Professor Richard Strohman at Berkeley who teaches a course in "Perspectives in Biology and Medicine" and is concerned with issues such as you described.

Occasionally, people get too "high" on high technology.

Gordon Edlin Professor of Genetics University of California Davis, CA 95616

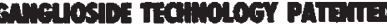

To the editor:

We have read with interest and also concern your article, "New Generation of Liposomes Stay in the Blood,"(Bio/Technology 6:1143, Oct. '88).

Dimitri Papahadjopoulos, speaking at the Fourth International Congress of Cell Biology, described experiments he and others conducted using liposomes containing the hydrophilic ganglioside GM-1 for the purpose of avoiding the reticuloendothelial system (RES) of the liver and spleen. This work was presented as a new development in the field. It is indeed a new development, but, in fact, the credit belongs to Technology Unlimited, Inc. of Wooster, $\mathrm{OH}$, who obtained U.S. Patent No. 4,501,728, "Masking of Liposomes from RES Recognition." The inventors (W. Blair Geho and John R. Lau) describe how to coat liposomes with sialic acid for the purpose of masking liposomes from the RES and thereby enhancing the in vivo half-life clearance.

It is of considerable importance to recognize that Papahadjopoulos and others have used our patented ganglioside technology to achieve their described effects. Ganglioside GM-1 contains, along with other sugars such as $\mathrm{N}$-acetyl galactosamine and $\mathrm{N}$ acetyl glucosamine, at least one mole of our RES avoidance molecule sialic acid (N-acetyl neuraminic acid).

This letter is an attempt to correct the record and place this significant invention in the proper context.

John R. Lau

Technology Unlimited, Inc. 1273 Lincoln Way West P. O. Box 723

Wooster, OH 44691

\section{anssmins modes}

To the editor:

We are grateful for the references $\checkmark$ to our chapter "Biological Modeling" (Computer Control of Fermentation Processes, in press at CRC Uniscience) in Douglas McCormick's article "Play with Purpose: Modeling Biotech Processes" (Bio/Technology 6:887, Aug. '88). We are afraid, though, that some readers might get the mistaken impression that the notion of classifying models as segregated or unsegregated originated with us. In fact, the classifications summarized by us in Figure 2 were proposed and discussed extensively by Tsuchiya et al. in 1966 (Adv. Chem. Eng. 6:125). We simply adopted their very useful classifications as part of our analysis of this important subject.

Robert Leipold and Prasad Dhurjati Dept. of Chemical Engineering University of Delaware Newark, DE 19716 